Supplementary Information for

\title{
Investigating the Uptake of Arsenate by Chlamydomonas reinhardtii Cells and its Effect on their Lipid Profile using Single Cell ICP-MS and Easy Ambient Sonic-Spray lonization - MS
}

\author{
Emmanouil Mavrakis ${ }^{a, \ddagger}$, Leonidas Mavroudakis ${ }^{a, \ddagger}$, Nikos Lydakis-Simantiris $^{b}$, Spiros A. \\ Pergantis $^{a, *}$ \\ a Environmental Chemical Processes Laboratory, Department of Chemistry, University of \\ Crete, Voutes Campus, Heraklion 70013, Greece \\ b Laboratory of Environmental Chemistry and of Biochemical Processes, Department of \\ Agriculture, Hellenic Mediterranean University, Chania 73133, Greece \\ *Corresponding Author e-mail: spergantis@uoc.gr
}

\section{Table of Contents:}

Figure S1. Step-by-step transformation of the intensity registered for a cell event into As mass.

Figure S2. ${ }^{75} \mathrm{As}^{+}$intensity distribution for control C. reinhardtii cells.

Figure S3. As mass distribution histograms and lognormal fits for unwashed $C$. reinhardtii cells.

Figure S4. Typical EASI-MS spectra of $C$. reinhardtii cells in positive and negative ion mode.

Figure S5. Microscopy images of C. reinhardtii before and after EASI-MS analysis.

Table S1. Precision data for technical replicates.

Table S2. Identified lipid molecules in C. reinhardtii cells. 
A Cell Detection Event

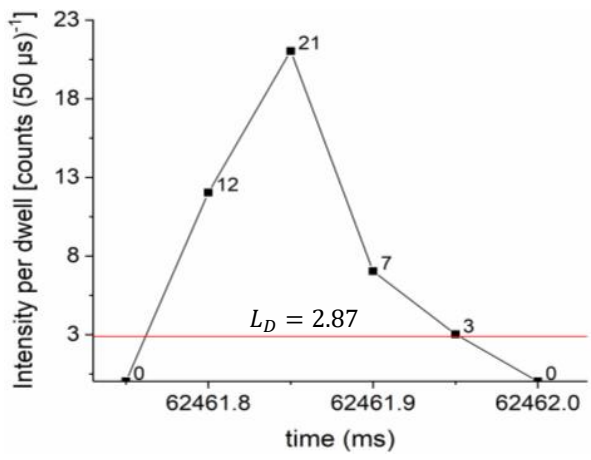

Peak Area $(P A)=\Sigma_{\text {Intensity per dwell }}$

$=12+21+7+3$

$=43$ counts $(1)$
B Calibration with ionic As standard

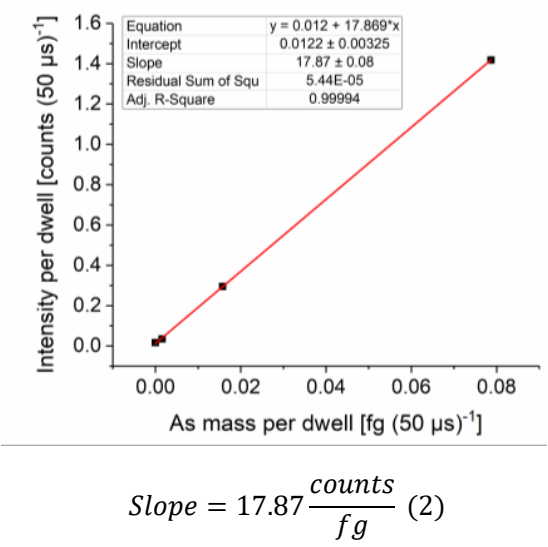

(1), (2) $\Rightarrow$ As mass per cell event $(f g)=\frac{P A}{\text { slope }}=\frac{43}{17.87}=2.41 \mathrm{fg}$

Figure S1. Step-by-step transformation of the intensity registered for the cell event shown in Panel A into As mass. This was achieved by summing the intensities of all 4 cell event points and converting their sum into As mass. This conversion was based on the ionic As mass flux calibration shown in Panel B. Numerical values and calculations are given below the two panels.
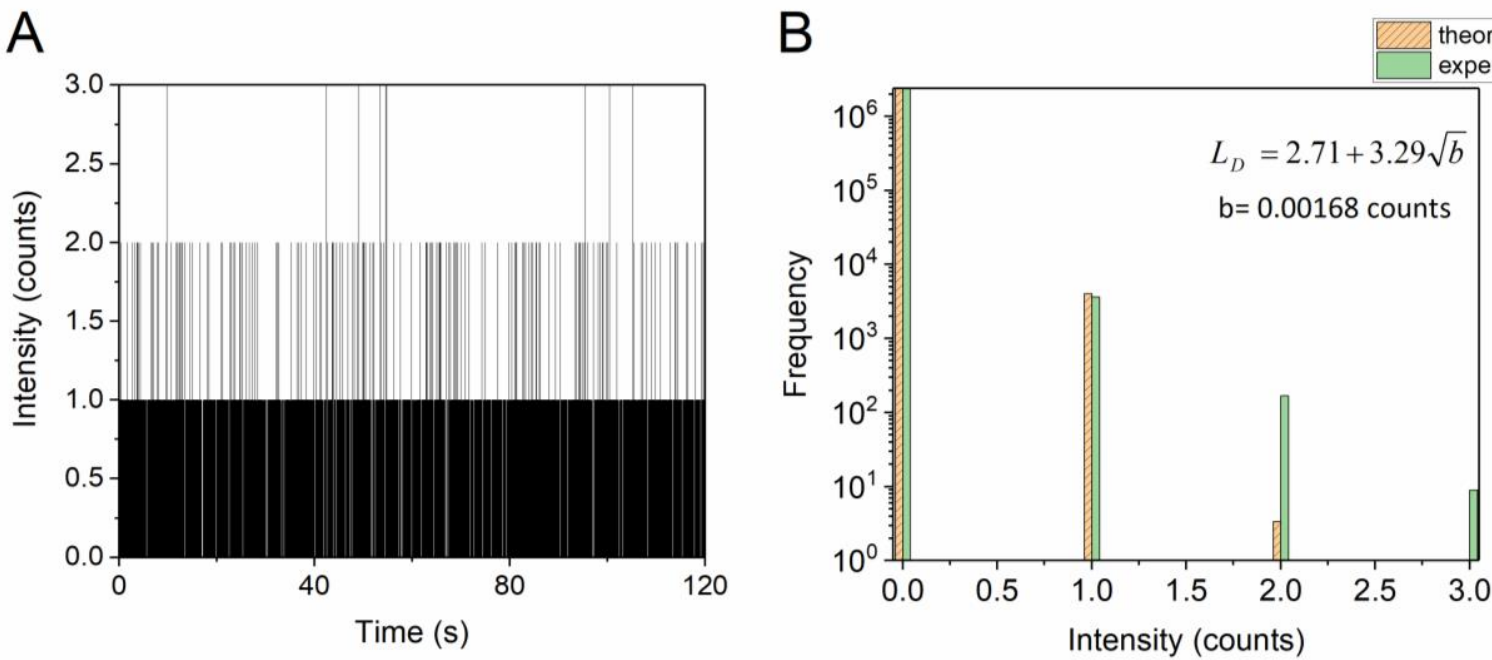

Figure S2. A) Time Resolved Acquisition (TRA) of ${ }^{75}$ As ${ }^{+}$for control C. reinhardtii cell suspension for 120 $s$ acquisition, B) Comparison of the plotted as histogram ${ }^{75}$ As intensity (experimental) with the corresponding (theoretical) Poisson distribution with a mean value b of 0.00168 . 
A
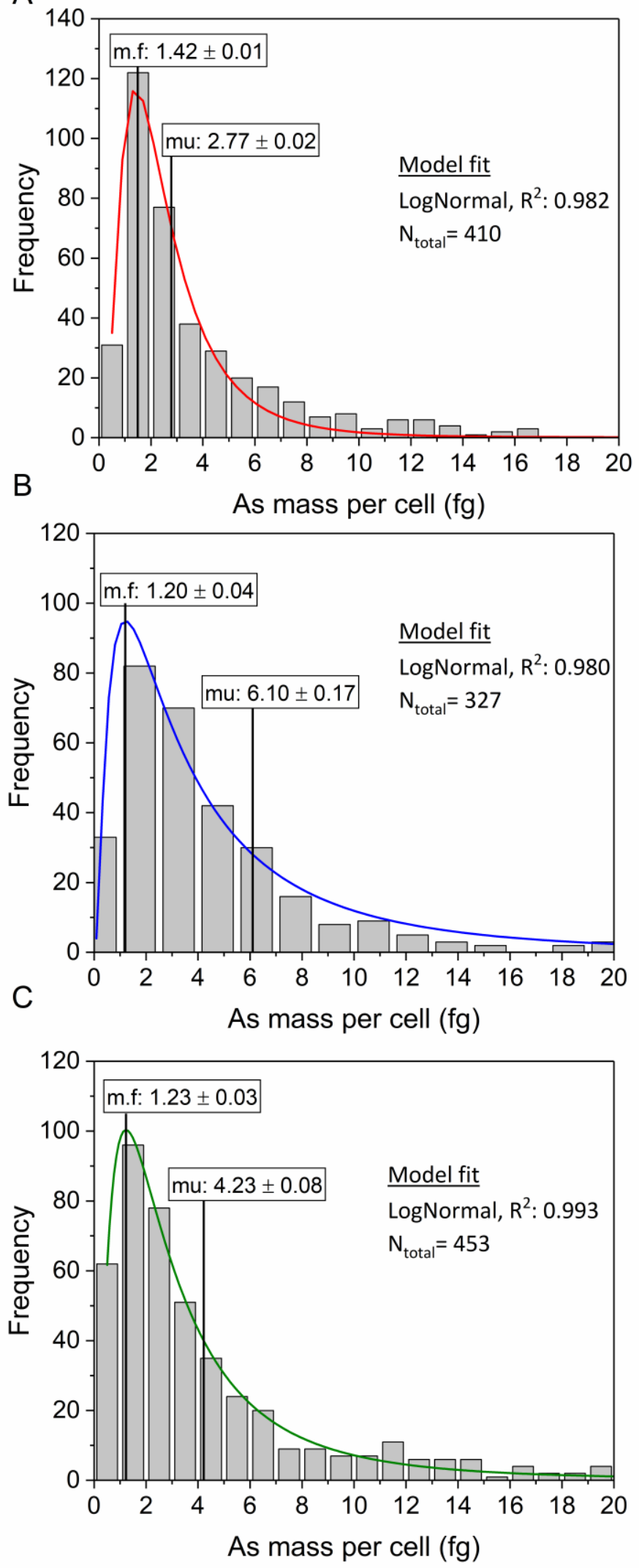

Figure S3. As mass distribution histograms for unwashed C. reinhardtii cells grown in 15 (A), 22.5 (B) and $30 \mu \mathrm{g} \mathrm{mL} \mathrm{L}^{-1} \mathrm{As}(\mathrm{C}) ; \mathrm{A}, \mathrm{C}$ binned at $1 \mathrm{fg}$ and $B$ at $1.5 \mathrm{fg}$. The lognormal probability function has been fitted to the mass histogram data in all three cases. Values for mu and m.f denote the lognormal mean and most frequently observed As mass per cell, respectively. 
A

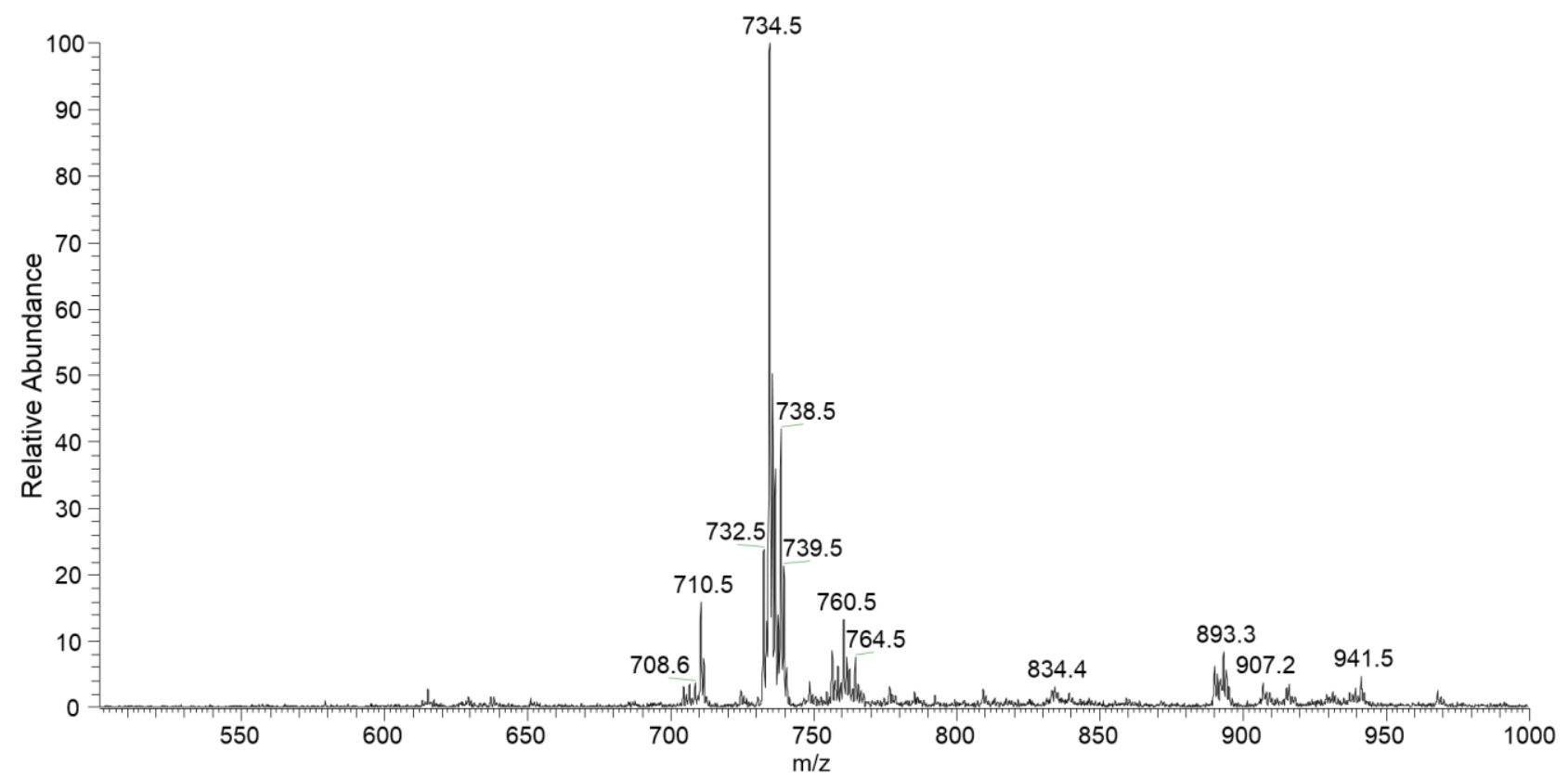

B

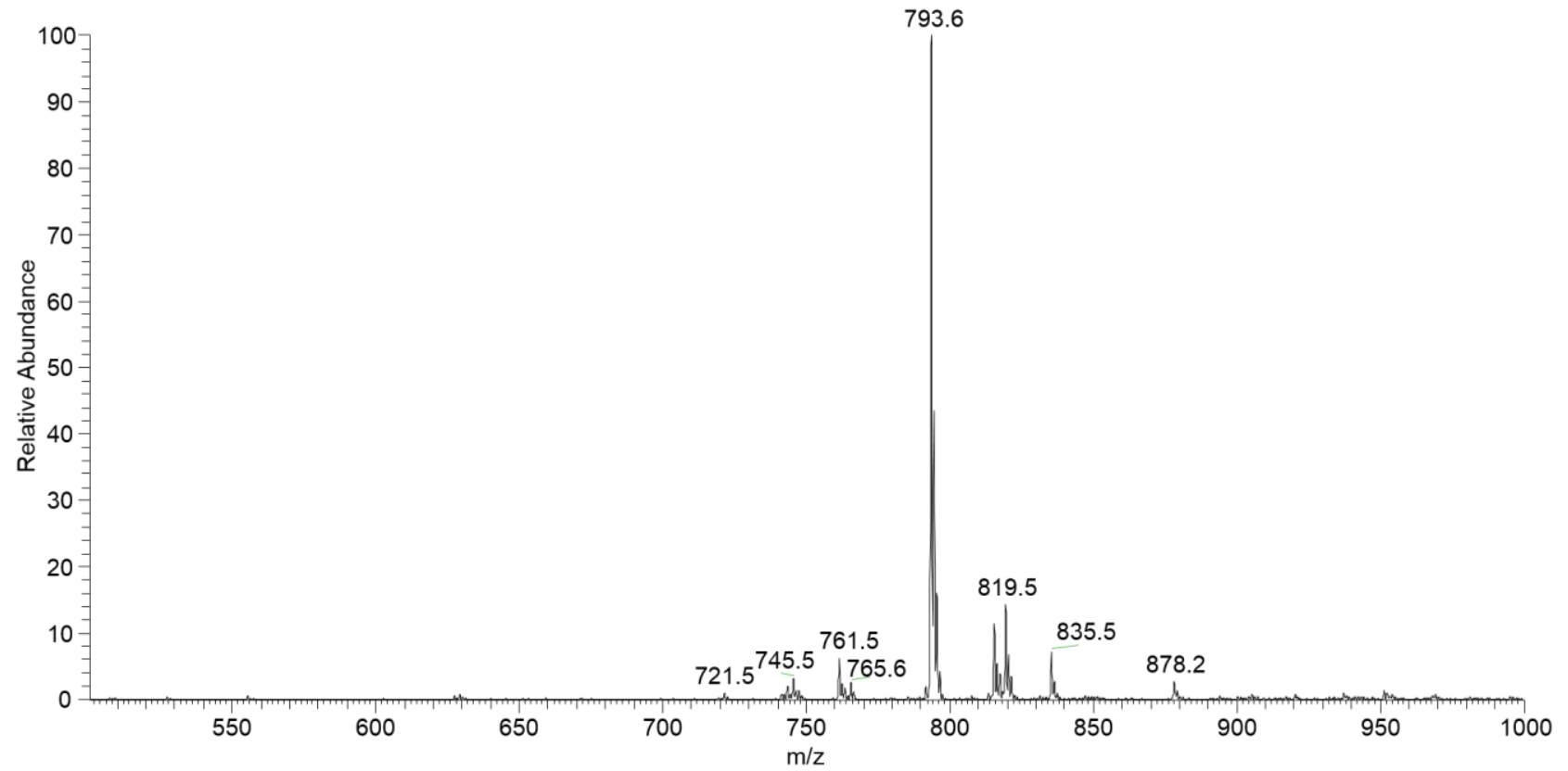

Figure S4. Typical positive (A) and negative (B) ion mode mass spectra obtained from the analysis of $C$. reinhardtii cells using EASI-MS. 


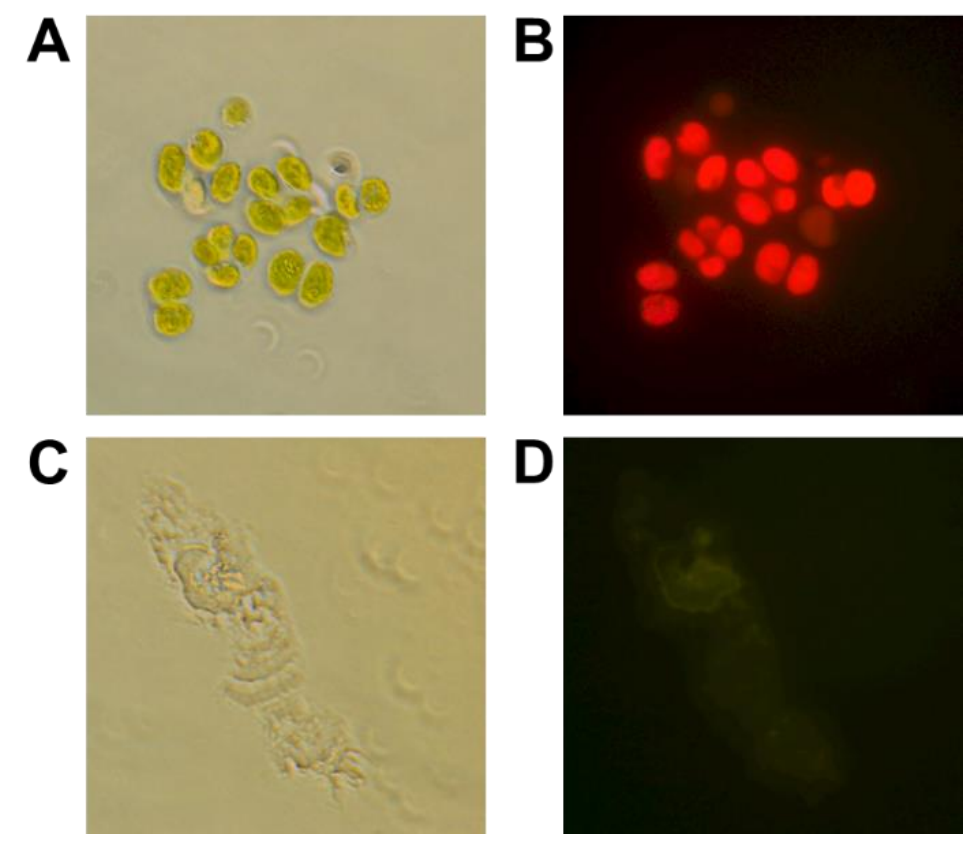

Figure S5. Optical (A) and fluorescence (B) microscopy images of $C$. reinhardtii cells that had been dried on a glass slide prior to EASI-MS analysis. Optical $(C)$ and fluorescence (D) microscopy images of cells that were sprayed with the spraying solvent and the aerosol formed was collected and resuspended in small volume of water. 
Table S1. Precision indicative data on the 3 independent technical replicates.

\begin{tabular}{|c|c|c|c|c|c|}
\hline Cell Samples & $\begin{array}{l}\text { Most } \\
\text { Freq. } \\
\text { Mass } \\
\text { (fg) }\end{array}$ & $\begin{array}{c}\text { Mean } \\
\text { Mass } \\
(f g)\end{array}$ & $\begin{array}{l}\text { No. of } \\
\text { Peaks }\end{array}$ & $\begin{array}{c}\text { Mean } \\
\text { Intensity } \\
\text { (counts) }\end{array}$ & $\begin{array}{l}\text { Dissolved } \\
\text { Intensity } \\
\text { (counts) }\end{array}$ \\
\hline Incubated in 15 ppm As Rep. 1 & 1.96 & 4.66 & 97 & 82.44 & $3.10 \mathrm{E}-03$ \\
\hline Incubated in 15 ppm As Rep. 2 & 2.16 & 4.63 & 99 & 81.80 & 3.03E-03 \\
\hline Incubated in 15 ppm As Rep. 3 & 2.16 & 4.27 & 90 & 75.38 & $3.06 \mathrm{E}-03$ \\
\hline Average & 2.09 & 4.52 & 95.3 & 79.87 & 3.06E-03 \\
\hline Stdev & 0.12 & 0.22 & 4.73 & 3.91 & $3.33 \mathrm{E}-05$ \\
\hline stdev\% & 5.53 & 4.82 & 4.96 & 4.89 & 1.09 \\
\hline Incubated in 22.5 ppm As Rep. 1 & 2.36 & 5.94 & 120 & 105.21 & 4.86E-03 \\
\hline Incubated in 22.5 ppm As Rep. 2 & 2.46 & 7.59 & 137 & 134.76 & $5.13 \mathrm{E}-03$ \\
\hline Incubated in 22.5 ppm As Rep. 3 & 2.46 & 6.55 & 95 & 116.05 & $5.08 \mathrm{E}-03$ \\
\hline Average & 2.42 & 6.69 & 117.3 & 118.67 & $5.02 E-03$ \\
\hline Stdev & 0.06 & 0.84 & 21.13 & 14.95 & 1.43E-04 \\
\hline stdev\% & 2.38 & 12.49 & 18.01 & 12.60 & 2.85 \\
\hline Incubated in 30 ppm As Rep. 1 & 2.16 & 4.46 & 104 & 78.78 & $3.85 E-03$ \\
\hline Incubated in 30 ppm As Rep. 2 & 2.31 & 4.84 & 83 & 79.98 & $3.90 \mathrm{E}-03$ \\
\hline Incubated in 30 ppm As Rep. 3 & 2.31 & 4.47 & 101 & 74.35 & 3.91E-03 \\
\hline Average & 2.26 & 4.59 & 96.0 & 77.70 & 3.89E-03 \\
\hline Stdev & 0.09 & 0.21 & 11.36 & 2.97 & 2.90E-05 \\
\hline stdev\% & 3.84 & 4.64 & 11.83 & 3.82 & 0.75 \\
\hline
\end{tabular}


Table S2. Annotations of lipids identified in C. reinhardtii cells.

\begin{tabular}{|c|c|c|c|}
\hline $\begin{array}{l}\text { Experimental } \\
\mathrm{m} / \mathrm{z}\end{array}$ & $\begin{array}{l}\text { Theoretical } \\
\mathbf{m} / \mathbf{z}\end{array}$ & Adduct & Identified Species \\
\hline 741.5 & 741.47 & {$[\mathrm{M}-\mathrm{H}]^{-}$} & PG(18:3/16:1) \\
\hline 743.5 & 743.49 & {$[\mathrm{M}-\mathrm{H}]^{-}$} & PG(18:3/16:0) \\
\hline 745.5 & 745.50 & {$[\mathrm{M}-\mathrm{H}]^{-}$} & PG(18:2/16:0) \\
\hline 747.5 & 747.52 & {$[\mathrm{M}-\mathrm{H}]^{-}$} & PG(18:1/16:0) \\
\hline 761.5 & 761.45 & {$[\mathrm{M}-\mathrm{H}]^{-}$} & SQDG(14:2/16:0) \\
\hline 791.5 & 791.50 & {$[\mathrm{M}-\mathrm{H}]^{-}$} & SQDG(16:0/16:1) \\
\hline 793.5 & 793.51 & {$[\mathrm{M}-\mathrm{H}]^{-}$} & SQDG(16:0/16:0) \\
\hline 815.5 & 815.50 & {$[\mathrm{M}-\mathrm{H}]^{-}$} & SQDG(18:3/16:0) \\
\hline 817.5 & 817.51 & {$[\mathrm{M}-\mathrm{H}]^{-}$} & SQDG(18:2/16:0) \\
\hline 819.5 & 819.53 & {$[\mathrm{M}-\mathrm{H}]^{-}$} & SQDG(18:1/16:0) \\
\hline 821.5 & 821.55 & {$[\mathrm{M}-\mathrm{H}]^{-}$} & SQDG(18:0/16:0) \\
\hline 831.5 & 831.53 & {$[\mathrm{M}-\mathrm{H}]^{-}$} & SQDG(19:2/16:0) \\
\hline 835.5 & 835.53 & {$[\mathrm{M}-\mathrm{H}]^{-}$} & $\mathrm{PI}(18: 1 / 16: 0)$ \\
\hline 847.4 & 847.56 & {$[\mathrm{M}-\mathrm{H}]^{-}$} & SQDG(20:1/16:0) \\
\hline 849.4 & 849.58 & {$[\mathrm{M}-\mathrm{H}]^{-}$} & SQDG(20:0/16:0) \\
\hline 474.5 & 474.38 & {$[\mathrm{M}+\mathrm{H}]^{+}$} & LysoDGTS(16:0) \\
\hline 496.4 & 496.36 & {$[\mathrm{M}+\mathrm{H}]^{+}$} & LysoDGTS(18:3) \\
\hline 704.5 & 704.55 & {$[\mathrm{M}+\mathrm{H}]^{+}$} & $\operatorname{DGTS}(32: 4)^{\mathrm{a}}$ \\
\hline 706.5 & 706.56 & {$[\mathrm{M}+\mathrm{H}]^{+}$} & $\operatorname{DGTS}(32: 3)^{\mathrm{b}}$ \\
\hline 708.5 & 708.58 & {$[\mathrm{M}+\mathrm{H}]^{+}$} & DGTS(16:0/16:2) \\
\hline 710.5 & 710.59 & {$[\mathrm{M}+\mathrm{H}]^{+}$} & DGTS(16:0/16:1) \\
\hline 732.5 & 732.58 & {$[\mathrm{M}+\mathrm{H}]^{+}$} & DGTS(16:0/18:4) \\
\hline 734.5 & 734.59 & {$[\mathrm{M}+\mathrm{H}]^{+}$} & DGTS(16:0/18:3) \\
\hline 736.5 & 736.61 & {$[\mathrm{M}+\mathrm{H}]^{+}$} & DGTS(16:0/18:2) \\
\hline 738.5 & 738.62 & {$[\mathrm{M}+\mathrm{H}]^{+}$} & DGTS(16:0/18:1) \\
\hline 748.5 & 748.61 & {$[\mathrm{M}+\mathrm{H}]^{+}$} & DGTS(16:0/19:3) \\
\hline 750.5 & 750.62 & {$[\mathrm{M}+\mathrm{H}]^{+}$} & DGTS(16:0/19:2) \\
\hline 754.5 & 754.56 & {$[\mathrm{M}+\mathrm{H}]^{+}$} & DGTS(18:3/18:4) \\
\hline 756.5 & 756.58 & {$[\mathrm{M}+\mathrm{H}]^{+}$} & $\operatorname{DGTS}(36: 6)^{\mathrm{c}}$ \\
\hline 758.5 & 758.59 & {$[\mathrm{M}+\mathrm{H}]^{+}$} & $\operatorname{DGTS}(36: 5)^{\mathrm{d}}$ \\
\hline 760.6 & 760.61 & {$[\mathrm{M}+\mathrm{H}]^{+}$} & $\operatorname{DGTS}(36: 4)^{\mathrm{e}}$ \\
\hline 762.5 & 762.62 & {$[\mathrm{M}+\mathrm{H}]^{+}$} & $\operatorname{DGTS}(36: 3)^{\mathrm{f}}$ \\
\hline 764.5 & 764.60 & {$\left[\mathrm{M}+\mathrm{H}+\mathrm{H}_{2} \mathrm{O}\right]^{+}$} & DGTS(16:0/19:4) \\
\hline 766.5 & 766.62 & {$\left[\mathrm{M}+\mathrm{H}+\mathrm{H}_{2} \mathrm{O}\right]^{+}$} & DGTS(16:0/19:3) \\
\hline 768.5 & 768.63 & {$\left[\mathrm{M}+\mathrm{H}+\mathrm{H}_{2} \mathrm{O}\right]^{+}$} & DGTS(16:0/19:2) \\
\hline 871.3 & 871.57 & {$[\mathrm{M}+\mathrm{H}]^{+}$} & Pheophytin a \\
\hline 893.3 & 893.54 & {$[\mathrm{M}+\mathrm{H}]^{+}$} & Chlorophyll a \\
\hline 907.2 & 907.51 & {$[\mathrm{M}+\mathrm{H}]^{+}$} & Chlorophyll b \\
\hline 915.5 & 915.53 & {$[\mathrm{M}+\mathrm{Na}]^{+}$} & Chlorophyll a \\
\hline 941.5 & 941.62 & {$[\mathrm{M}+\mathrm{Na}]^{+}$} & DGDG(18:1/16:0) \\
\hline
\end{tabular}

${ }^{a}$ Mixture of isomers DGTS(16:0/16:4), DGTS(16:1/16:3), DGTS(14:0/18:4)

${ }^{b}$ Mixture of isomers DGTS(16:0/16:3), DGTS(14:0/18:3)

${ }^{c}$ Mixture of isomers DGTS(18:2/18:4), DGTS(18:3/18:3)

${ }^{\mathrm{d}}$ Mixture of isomers DGTS(18:1/18:4), DGTS(18:2/18:3)

eMixture of isomers DGTS(18:0/18:4), DGTS(18:1/18:3), DGTS(18:2/18:2)

fMixture of isomers DGTS(18:0/18:3), DGTS(18:1/18:2) 